\title{
Some variables to discuss regarding vesicoureteral reflux management
}

\author{
Sergio Hernando Mina-Riascos ${ }^{1,2} \cdot$ Nicolas Fernandez ${ }^{3} \cdot$ Herney Andrés García-Perdomo ${ }^{1,2}$ (D)
}

Received: 25 July 2021 / Revised: 25 July 2021 / Accepted: 27 July 2021 / Published online: 6 August 2021

(c) The Author(s), under exclusive licence to Springer-Verlag GmbH Germany, part of Springer Nature 2021

We appreciate the comments from Selvi et al. They are valuable for an updated discussion.

The understanding of Vesicoureteral reflux (VUR) management has evolved over the last decades [1]. Regarding the interventions, our study showed that there were no differences in urinary tract infection (UTI) episodes after VUR correction in patients undergoing endoscopic management compared with vesicoureteral reimplantation. Also, we described that our results were limited due to the lack of stratification of grade, patient's age, and the lack of information regarding bladder and bowel dysfunction [2].

Additionally, the EAU-ESPU guideline recommendations for daytime lower urinary tract conditions in children refer that voiding-phase dysfunctions might be more important for the existence of UTI than VUR itself. Nonetheless, they did not inform about the important topics that you have been described in this letter.

Regarding your point of view, we agree that the interpretation of the voiding cystourethrography (VCUG) has additional variables that might improve its predictive performance in clinical settings. Nonetheless, the lack of a standardized protocol makes VCUG studies difficult to be reproducible. This factor also limited our analysis. The only way such an issue can be overcome is with large-scale prospective standardized VCUG protocols. New variables such as the distal diameter of the ureter, the moment when reflux was first detected, and whether it was on the first or second cycle will also be important variables to include in this prospective collaborative study.

As an additional note, we are trying contrast-enhanced ultrasonography to study vesicoureteral reflux and avoid harmful exposure to radiation at our institution.

Again, we appreciate this opportunity to discuss essential variables that might affect the outcomes in our patients.

\section{Declarations}

Conflict of interest The authors declare no competing interests.

\section{References}

1. Fernandez N, Puerto A, Azuero A, Hannick M, Rickard M (2018) Historical bibliometric analysis of the top cited articles on vesicoureteral reflux 1950-2016, and incorporation of an impact index. J Pediatr Urol 14(5):446.e1-446.e9

2. Mina-Riascos SH, Fernández N, García-Perdomo HA (2021) Effectiveness and risks of endoscopic management compared to vesicoureteral reimplantation in patients with high-grade vesicoureteral reflux: systematic review and network meta-analysis. Eur J Pediatr 180(5):1383-1391

Publisher's Note Springer Nature remains neutral with regard to jurisdictional claims in published maps and institutional affiliations.
Communicated by Peter de Winter

Herney Andrés García-Perdomo

herney.garcia@correounivalle.edu.co

1 Department of Surgery/Urology, School of Medicine, Universidad del Valle, Cali, Colombia

2 UROGIV Research Group, School of Medicine, Universidad del Valle, Cali, Colombia

3 Division of Urology, Seattle Children's Hospital, The University of Washington, Seattle, WA, USA 